\title{
Hardware Design Improvements to the Major Constituent Analyzer
}

Scott Combs, Daniel Schwietert, Marcial Anaya, Shannon DeWolf, Dave Merrill, Ben D. Gardner \& Souzan Thoresen

Hamilton Sundstrand Space Systems International

John Granahan, Paul Belcher

The Boeing Company

Chris Matty

NASA

\section{ABSTRACT:}

The Major Constituent Analyzer (MCA) onboard the International Space Station (ISS) is designed to monitor the major constituents of the ISS's internal atmosphere. This mass spectrometer based system is an integral part of the Environmental Control and Life Support System (ECLSS) and is a primary tool for the management of ISS atmosphere composition.

As a part of NASA Change Request CR10773A, several alterations to the hardware have been made to accommodate improved MCA logistics. First, the ORU 08 verification gas assembly has been modified to allow the verification gas cylinder to be installed on orbit. The verification gas is an essential MCA consumable that requires periodic replenishment. Designing the cylinder for subassembly transport reduces the size and weight of the maintained item for launch. The redesign of the ORU 08 assembly includes a redesigned housing, cylinder mounting apparatus, and pneumatic connection.

The second hardware change is a redesigned wiring harness for the ORU 02 analyzer. The ORU 02 electrical connector interface was damaged in a previous on-orbit installation, and this necessitated the development of a temporary fix while a more permanent solution was developed. The new wiring harness design includes flexible cable as well as indexing fasteners and guide-pins, and provides better accessibility during the on-orbit maintenance operation.

This presentation will describe the hardware improvements being implemented for MCA as well as the expected improvement to logistics and maintenance. 


\title{
Hardware Design Improvements to the Major Constituent Analyzer
}

\author{
Scott Combs ${ }^{1}$, Daniel Schwietert ${ }^{2}$, Marcial Anaya ${ }^{3}$, Dave Merrill ${ }^{4}$, \\ Souzan Maleki Thoresen ${ }^{5}$ and Ben D. Gardner ${ }^{6}$ \\ Hamilton Sundstrand Corporation, Pomona, California, 91767 \\ John Granahan $^{7}$ and Paul Belcher ${ }^{8}$ \\ The Boeing Company, Houston, Texas, 77059 \\ and \\ Chris Matty ${ }^{9}$ \\ NASA Johnson Space Center, Houston, Texas, 77058
}

\begin{abstract}
The Major Constituent Analyzer (MCA) onboard the International Space Station (ISS) is a mass spectrometry-based system that is an integral part of the Environmental Control and Life Support System (ECLSS) and a primary tool for the management of ISS atmosphere composition. As part of NASA Change Request CR107733A, several hardware improvements have been made to accommodate better MCA logistics. First, ORU 02, the MCA's sensor core, has been modified both with a more flexible and accessible wiring harness for easier on-obit maintenance while the ion pump voltage and gas flow rate have been reduced to increase ion pump lifetime. A second module, the verification gas assembly (ORU 08), has been redesigned to allow for separate launch of the verification gas cylinder subassembly, reducing size and weight during launch. These design improvements, along with an upcoming firmware revision, are intended to reduce the logistical effort supporting the MCA and decrease the spares requirement through the ISS lifetime. Analysis shows that implementation of these improvements should reduce the on-orbit spares need from 8 ORU 02s and 3 ORU 08s, to 5 ORU 02s and 2 ORU 08s through year 2020.This presentation describes these hardware modifications and the expected improvement to logistics and maintenance.
\end{abstract}

$\begin{array}{ll}\text { ISS } & =\text { International Space Station } \\ \text { MCA } & =\text { Major Constituent Analyzer } \\ \text { ORU } & =\text { Orbit Replaceable Unit }\end{array}$

\section{Nomenclature}

${ }^{1}$ Project Engineer, Hamilton Sundstrand, 2771 North Garey Ave., Pomona, CA 91767.

${ }^{2}$ Mechanical Engineer, Hamilton Sundstrand, 2771 North Garey Ave., Pomona, CA 91767.

${ }^{3}$ Mechanical Engineer, Hamilton Sundstrand, 2771 North Garey Ave., Pomona, CA 91767.

${ }^{4}$ Engineering Manager, Hamilton Sundstrand, 2771 North Garey Ave., Pomona, CA 91767.

${ }^{5}$ Program Manager, Hamilton Sundstrand, 2771 North Garey Ave., Pomona, CA 91767.

${ }^{6}$ Project Engineer, Hamilton Sundstrand, 2771 North Garey Ave., Pomona, CA 91767.

${ }^{7}$ Mechanical Systems Design and Analysis Engineer, Environmental Control and Life Support Systems, The Boeing Company, 13100 Space Center Boulevard, Houston Texas. 77059/HB2-40, AIAA Associate Fellow.

${ }^{8}$ Mechanical Systems Design and Analysis Engineer, Environmental Control and Life Support Systems, The Boeing Company, 13100 Space Center Boulevard, Mail Code HB2-40, Houston Texas. 77059.

${ }^{9}$ Atmosphere Revitalization Subsystem Manager, EC6/Flight Program Support Branch, NASA Johnson Space Center, 2101 NASA Parkway, Houston, Texas 77058. 


\section{Introduction}

$\mathrm{T}$ HE MCA is a mass spectrometer-based instrument on-board the ISS that serves as the primary monitoring tool for six major atmospheric constituents ${ }^{1-4}$. This monitoring is critical to ensure crew safety and to verify safe operation of the Atmosphere Control System (ACS), the Atmosphere Revitalization (AR) system, and Airlock Extravehicular Activity (EVA) preparation. The major constituents (nitrogen $\left(\mathrm{N}_{2}\right)$, oxygen $\left(\mathrm{O}_{2}\right)$, hydrogen $\left(\mathrm{H}_{2}\right)$, carbon dioxide $\left(\mathrm{CO}_{2}\right)$, methane $\left(\mathrm{CH}_{4}\right)$, and water vapor $\left.\left(\mathrm{H}_{2} \mathrm{O}\right)\right)$, are monitored under the control of the ISS Internal Systems (INTSYS) software using samples drawn from various modules of the ISS through a network of sample lines. A continuous readout of the partial pressures for each of these gases is available at all times to the crew and ground control.

The MCA is modularly designed as a combination of seven On-orbit Replaceable Units (ORUs), shown in Figure 1, to facilitate maintenance operations (ORU 03 was proposed but not included in the final design). The modular design minimizes the logistical effort of supporting MCA by precluding the need to replace the entire system when periodic maintenance is required. Thus, logistical support is more flexible, on-orbit installation time is conserved, and launch and refurbishment costs are minimized. The imminent retirement of the space shuttle has generated interest in further optimizing MCA logistics due to the expected reduction in both launch and down-mass opportunity. Several improvements are being made to the MCA in order to increase the replacement interval of the ORUs and to ease the transport and installation effort as a part of NASA Change Request CR10773A. Typically, ORU 05 - sample pumps, ORU 08 - verification gas assembly, and ORU 02 - mass spectrometer assembly, require periodic replacement due to wear and depletion of consumables, while the EEPROMs of ORU 01 - data and control assembly, require infrequent refresh. The ORU 05 design will remain unchanged, and the ORU 01 EEPROMS are being recycled in coordination with a firmware upgrade that is also part of the logistical optimization and will be described elsewhere. Presented here are the improvements being implemented to ORU 08 and ORU 02, and the resulting improvement to the MCA ORU traffic model that is consequently expected.

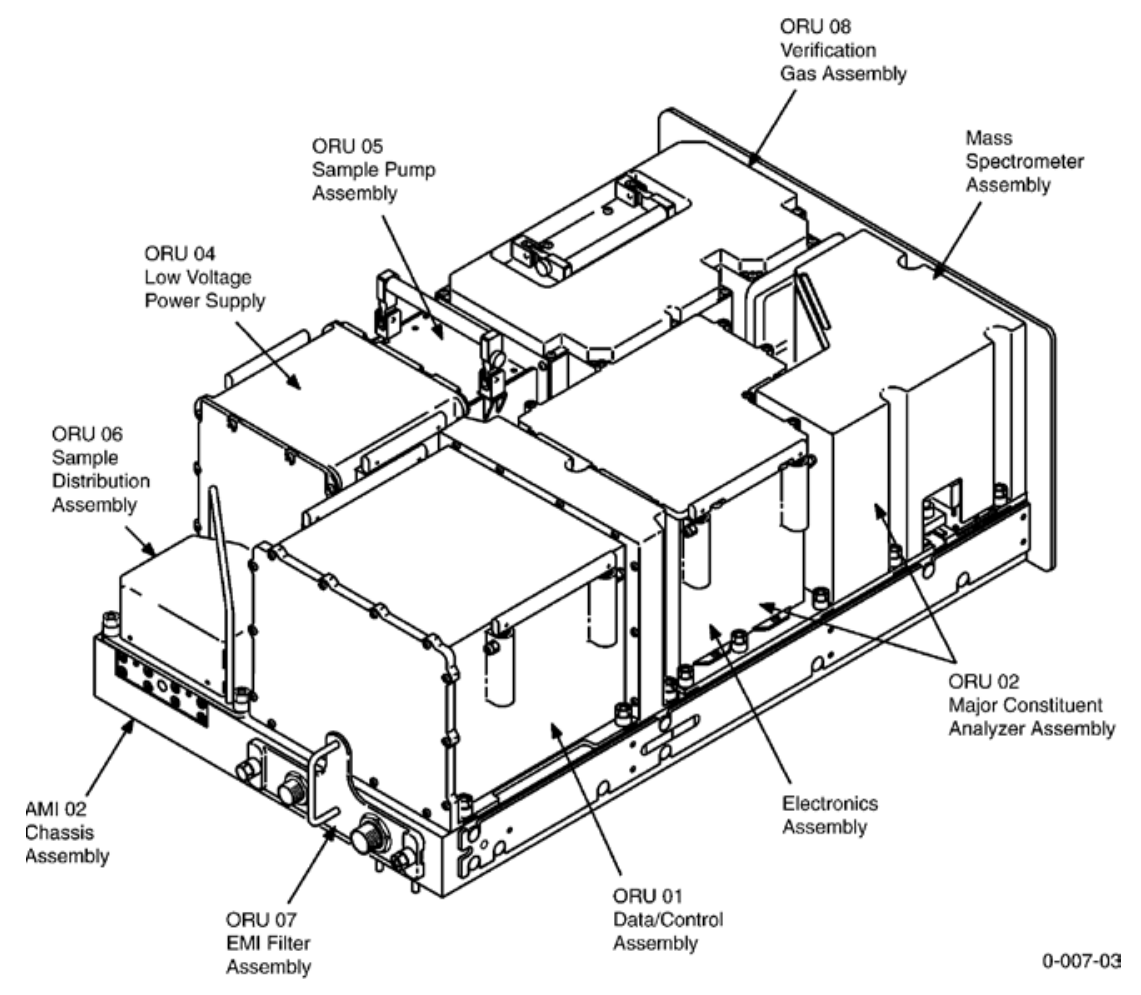

Figure 1: The MCA.

\section{ORU 08}

ORU 08, the Verification Gas Assembly, supplies the gas used periodically to calibrate the MCA. The verification gas is a precisely known mixture of $\mathrm{N}_{2}, \mathrm{O}_{2}, \mathrm{CO}_{2}, \mathrm{H}_{2}, \mathrm{CH}_{4}$ and $\mathrm{CD}_{4}$ (deuterated methane) at volume 
concentrations similar to the major constituents found on-board ISS. $\mathrm{CD}_{4}$ is used in place of $\mathrm{H}_{2} \mathrm{O}$ in order to avoid the condensation and adsorption/desorption phenomena inherent to the transport of pressurized $\mathrm{H}_{2} \mathrm{O}_{\text {(v) }}$ samples through the pneumatic lines. The verification gas pressure vessel is initially pressurized to $1000 \mathrm{psi}$ and provides enough verification gas for approximately three years of MCA calibrations and ancillary activities before requiring replacement.

The original ORU 08 design, shown in Figure 2, includes the pressurized verification gas cylinder, along with plumbing hardware, valves, pressure transducer, and pressure regulator, integrated into an impact resistant housing that directly interfaces to the MCA chassis frame. Typically, a fully charged ORU 08 is launched independently and installed into the MCA on orbit by replacing the depleted ORU 08. However, it is desirable to reduce the size and weight of the ORU 08 package for future launches. As a result, a new design for ORU 08 has been developed that allows the verification gas cylinder to be installed into the ORU on orbit as a subassembly of the ORU 08 , while the remaining components are already attached to the MCA chassis. The redesigned ORU 08 is shown in Figure 3 . The subassembly is fully transportable in a protected housing suitable for launch environments.

The redesigned ORU 08 assembly includes the original housing, and a revised cylinder mounting apparatus and pneumatic connection.

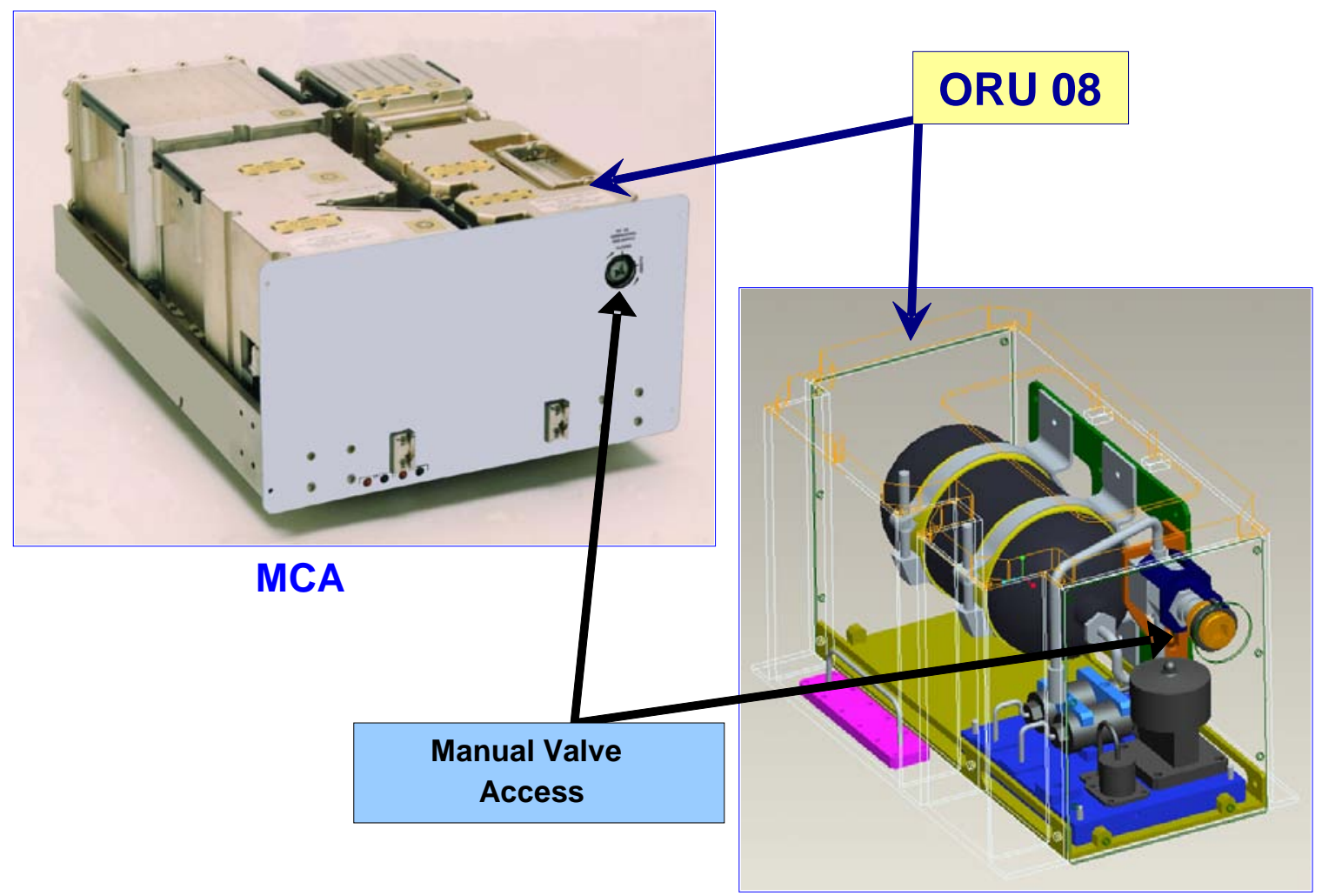

Figure 2: MCA and the current configuration of the ORU 08 assembly. 


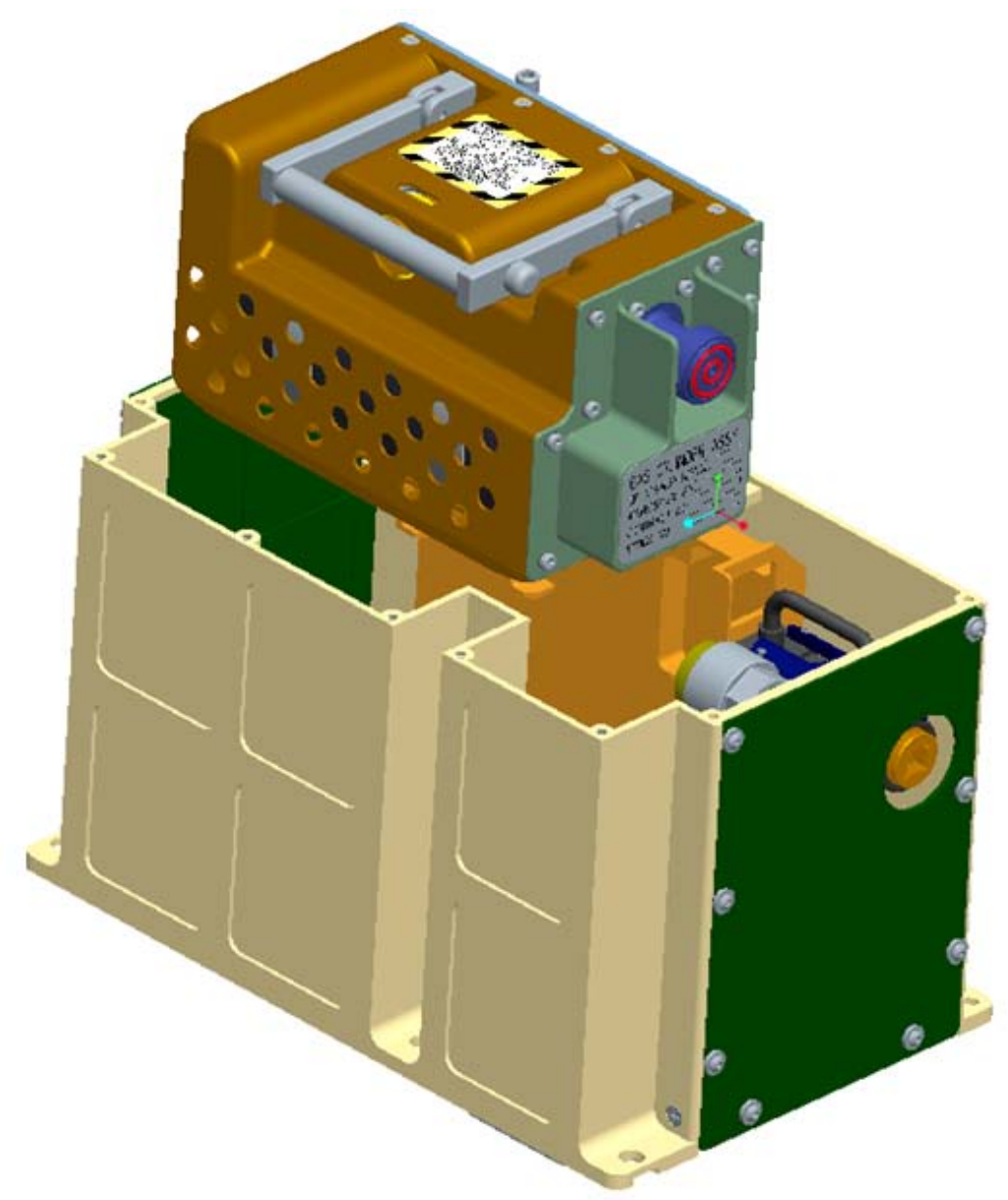

Figure 3: The revised ORU 08 design. The verification gas subassembly is replaceable within the ORU 08 housing and has a pneumatic connector that simplifies the attachment process.

Existing ORU 08s are being refurbished to the new design and two additional ORU 08s of the new design are being constructed as part of the PIO 89 program. The new design increases the overall weight of the ORU 08 from $12 \mathrm{lbs}$ to $15.5 \mathrm{lbs}$. However, this increased weight is a factor only for the initial launch of the new ORU 08s for installation into the two MCAs now onboard the ISS. The verification gas subassembly weighs only $5.4 \mathrm{lbs}$, which results in a launch weight decrease of $6.6 \mathrm{lbs}$ for all subsequent launches. Additionally, the shipping volume is decreased from $675 \mathrm{in}^{3}$ for the original ORU 08 to $230 \mathrm{in}^{3}$ for the subassembly. It is anticipated that the lower weight and volume will both reduce the launch costs and increase the compatibility with future launch vehicles that may have limited payload capability.

\section{ORU 02}

The ORU 02 Mass Spectrometer Assembly forms the core of the MCA technology. Periodically ORU 02 is replaced and refurbished due to time-dependent wear of the ion source and the ion pump. Part of the replacement process involves connecting a wiring harness from the ORU 02 to the MCA chassis. During R\&R of an ORU 02 in 2006, the electrical connector on the MCA chassis became bent and could not be mated with the ORU 02 connector. An on-orbit modification was made to the ORU 02 harness assembly to allow temporary connection and keep the MCA operational.

Since then, a more permanent connector saver solution has been developed to stabilize the connector in the MCA chassis assembly and provide a mating surface to which all subsequent ORU 02s will interface. The connector saver was designed based on analyses of detailed photographs that showed the connector damage. The design is intended to provide a connecting mechanism that compensates for the permanent damage on the MCA chassis, is easy to install, and reduces the likelihood of further damage to the MCA. Four variants of the connector saver have been developed to account for the presence of both original and retrofitted ORU 02s, as well as the fact that there are now 
two MCAs on orbit and only one of them has a chassis with a damaged electrical connector. The new design, shown in Figure 4, includes indexing fasteners and guide pins to aid in mounting the ORU 02 wiring harness connectors to either of the new chassis connector savers.

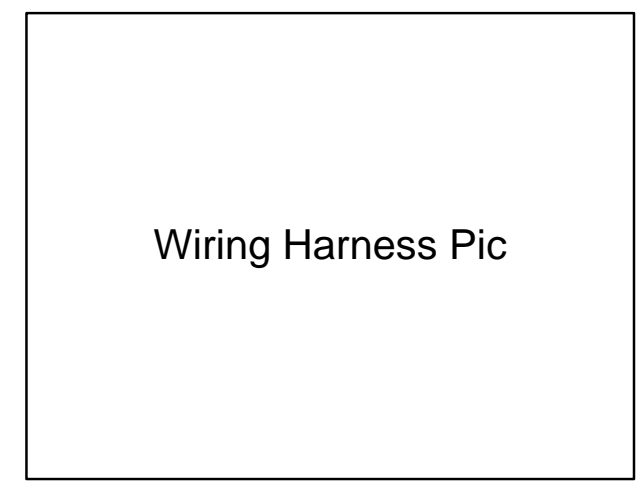

Figure 4: The ORU 02 wiring harness assembly.

Paragraph on the reduced ion pump voltage.

\section{Logistical Support}

The anticipated need for additional ORUs is currently under evaluation. In the traffic model currently being used by the MCA program, several identical ORUs are cycled as on orbit active, on-orbit spare, flight ready spare and unit under refurbishment. With the space shuttle retiring in the summer of 2011, however, it is no longer possible to down-manifest ORUs for refurbishment. Consequently, additional ORUs are being manufactured. The current and projected traffic models for ORU 02 and 08 are shown in Figure 5. The current model anticipates that, based on past performance, an ORU 02 will last for approximately 1 year and that an ORU 08 will last for 3 years. The improvements being implemented as part of CR10773A are predicted to increase the lifetimes of ORU 02s to at least 1.5 years and ORU 08s to 4 years under full MCA utilization. A second MCA is now on orbit and is expected to be functional beginning in 2012. Assuming that this MCA is used at a 50\% duty cycle, the expected lifetimes for the ORUs used on that system should be double. 
Current ORU Usage

\begin{tabular}{|c|c|c|c|c|c|c|c|c|c|c|c|c|c|c|c|c|c|c|}
\hline \multirow[b]{2}{*}{ ORU 02} & & & & & & & & & & \multirow[b]{2}{*}{$1 y$} & \multirow{2}{*}{\multicolumn{2}{|c|}{$1 \mathrm{y}$}} & \multirow[b]{2}{*}{$1 \mathrm{y}$} & \multirow{2}{*}{$1 y$} & \multirow{2}{*}{\multicolumn{2}{|c|}{\begin{tabular}{|l|l|}
1 & \\
19 & $1 \times 1$ \\
\end{tabular}}} & \multirow{2}{*}{\multicolumn{2}{|c|}{ T19 }} \\
\hline & \begin{tabular}{|l|l|}
$1 y$ & 1 \\
\end{tabular} & \begin{tabular}{l|l}
$y$ & $1 y$
\end{tabular} & $1 \mathrm{y}$ & $1 \mathrm{y}$ & $1 y$ & $1 y$ & $1 \mathrm{y}$ & $1 \mathrm{y}$ & $1 y$ & & & & & & & & & \\
\hline $\mathrm{RU} C$ & $1.5 \mathrm{y}$ & & $3 y$ & & 3 & & & & $3 y$ & & & $3 y$ & & & & $3 y$ & & $.5 \mathrm{y}$ \\
\hline
\end{tabular}

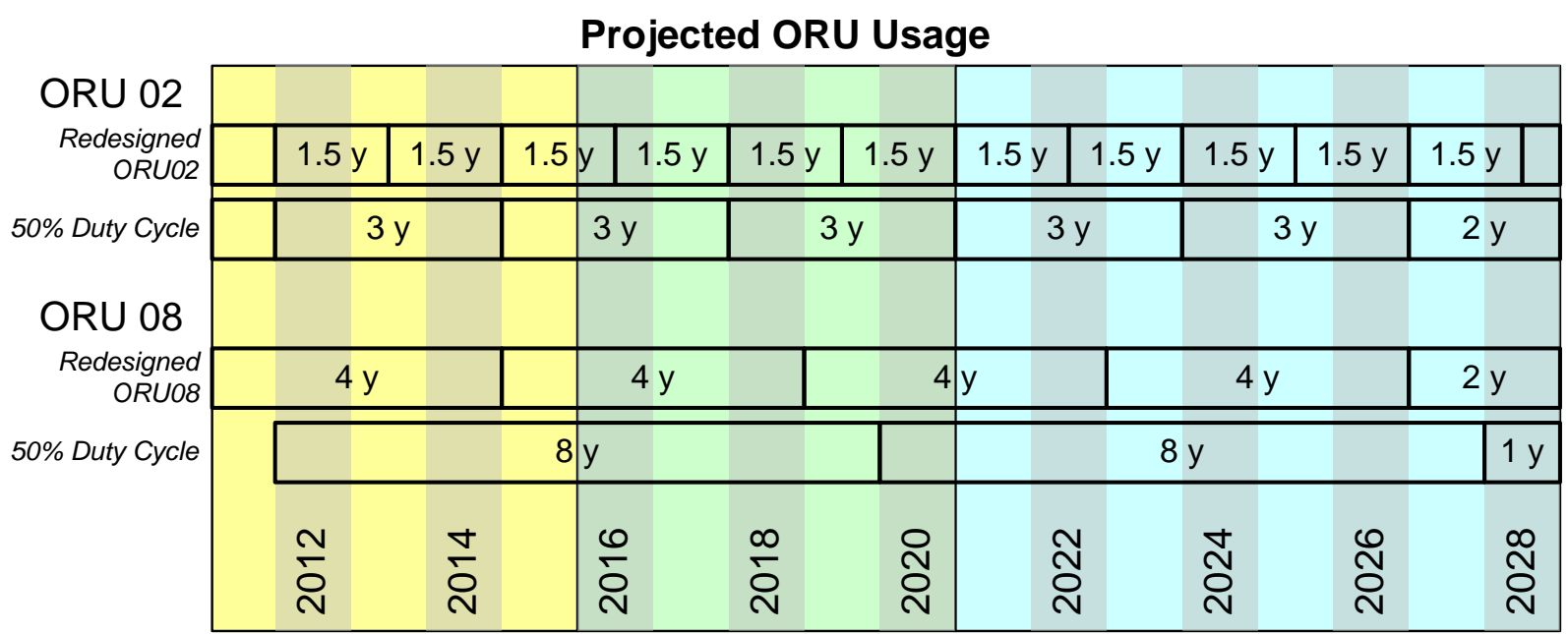

Figure 5: Anticipated utilization of ORU 02s and ORU 08s through 2015, 2020, and 2028.

The anticipated number of ORU 02s and 08s needed for MCA support under the best-case projected ORU usage traffic model are listed in Table 1. The PIO 89 program is currently building 4 ORU 02s and 2 ORU 08s for delivery by 2013. These, along with 2 existing ORU 02s and an ORU 08 being refurbished will ensure that MCA has adequate spares through 2015. However, supporting MCA to 2020 and beyond will require the manufacture of additional ORUs or the creation of down-manifest capability so that existing ORUs can be refurbished and returned to the logistics traffic stream.

\begin{tabular}{|l|c|c|c|}
\hline ORU\Date & 2015 & 2020 & 2028 \\
\hline ORU 02 & 5 & 9 & 18 \\
\hline ORU 08 & 3 & 5 & 8 \\
\hline
\end{tabular}

Table 1: ORU 02 and ORU 08 requirements required over ISS lifetime.

It should be noted that the other ORUs have also been evaluated for support of the MCA lifetime. ORU 04s - the low voltage power supply, ORU 06s - the sample distribution assembly, and ORU 07s - the EMI filter, each have a low probability of failure and are expected to last the lifetime of the MCA. There is a spare of each if needed on orbit. ORU 05 - the sample pump assembly, contains redundant pumps, each of which has a relatively long (3-5 year) expected lifetime. The current three spare units are being augmented with one additional unit being built under PIO 89. Finally, ORU 01 - the data/control assembly, requires an EEPROM refresh every 10 years. Two of the three existing ORU 01s have been refreshed and will not require further refresh until 2020. The third ORU 01 currently on orbit already requires an EEPROM refresh, and this is expected to occur once this ORU 01 is replaced with one containing updated firmware, being developed under CR10773A. Logistically it should not be necessary to build an additional ORU 01 provided that existing ORU 01s can be down-manifested before 2020. However, it is possible that a partial ORU 01 redesign and build may be required nonetheless due to EEPROM obsolescence.

\section{Conclusion}

The MCA is an integral part of ISS ECLSS with demonstrated utility for life support on ISS. Continued logistical support in the face of shuttle retirement requires that opportunities for further optimizing MCA design for performance and logistics support be implemented. The improvements being made to ORU 02 and ORU 08 design, 
in combination with the firmware improvements currently underway, will help increase the lifetime of these ORUs and reduce the effort required to support MCA.

\section{References}

${ }^{1}$ Gardner, B. D., Erwin, P. M., Thoresen, S. M., Granahan, J., and Matty, C., “International Space Station Major Constituent Analyzer On-orbit Performance," 40 ${ }^{\text {th }}$ International Conference on Environmental Systems, Barcelona, Spain 2010.

${ }^{2}$ Gardner, B. D., Erwin, P. M., Lee, W. T., Tissandier, A. M. and Thoresen, S. M., "Improving the measurement Accuracy of Water Partial Pressure Using the Major Constituent Analyzer," $39^{\text {th }}$ International Conference on Environmental Systems, Savannah, GA 2009.

${ }^{3}$ Thoresen, S. M., Steiner, G. and Granahan, J., “International Space Station (ISS) Major Constituent Analyzer (MCA) Onorbit Performance,” $38^{\text {th }}$ International Conference on Environmental Systems, San Francisco, CA 2008.

${ }^{4}$ Steiner, G., Thoresen, S. M., Reysa, R. and Granahan, J., "International Space Station (ISS) Major Constituent Analyzer (MCA) On-orbit Performance,” 36 ${ }^{\text {th }}$ International Conference on Environmental Systems, Norfolk, VA 2006. 